\title{
A generalization of Diaz-Margolis's fixed point theorem and its application to the stability of generalized Volterra integral equations
}

\section{Wei-Shih Du*}

"Correspondence:

wsdu@mail.nknu.edu.tw Department of Mathematics, National Kaohsiung Normal University, Kaohsiung, 82444, Taiwan

\begin{abstract}
In this paper, a generalization of Diaz-Margolis's fixed point theorem is established. As applications of the generalized Diaz-Margolis's fixed point theorem, we present some existence theorems of the Hyers-Ulam stability for a general class of the nonlinear Volterra integral equations in Banach spaces.
\end{abstract}

MSC: 26D10; 34K20; 45D05; 47H10

Keywords: Hyers-Ulam stability; nonlinear Volterra integral equation; $\mathcal{M T}$-function $(\mathcal{R}$-function); generalized metric space; Diaz-Margolis's fixed point theorem

\section{Introduction and preliminaries}

The stability of functional equations was originally raised in a famous talk given by Ulam [1] at Wisconsin University in 1940. The problem posed by Ulam was the following:

Let $G_{1}$ be a group and let $G_{2}$ be a metric group with the metric d. Given $\varepsilon>0$, does there exist $a \delta>0$ such that if a function $h: G_{1} \rightarrow G_{2}$ satisfies the inequality

$$
d(h(x y), h(x) h(y))<\delta
$$

for all $x, y \in G_{1}$, then there exists a homomorphism $H: G_{1} \rightarrow G_{2}$ with

$$
d(h(x), H(x))<\varepsilon
$$

for all $x \in G_{1}$ ?

A partial answer to Ulam's question in the case of Banach spaces was given by Hyers [2] in 1941. Later, Aoki [3] studied this problem for additive mappings and Rassias [4] generalized Hyers' theorem for the stability of unbounded Cauchy equations. Since then the rapid growth of the study of stability of functional equations has been developed at a high rate by several authors in the last decades; for more details, we refer the readers to [3-14] and references therein.

Let $(E,\|\cdot\|)$ be a normed space over a field $\mathcal{K}$ (either $\mathbb{R}$ or $\mathbb{C}$ ), $I=[a, b]$ be a closed interval in $\mathbb{R}$ and $c \in I$. Let $G: I \times I \times E \rightarrow E, s: I \rightarrow \mathcal{K}$ and $\kappa: I \rightarrow E$ be mappings. In this paper,

(c) 2015 Du. This article is distributed under the terms of the Creative Commons Attribution 4.0 International License (http://creativecommons.org/licenses/by/4.0/), which permits unrestricted use, distribution, and reproduction in any medium, provided you give appropriate credit to the original author(s) and the source, provide a link to the Creative Commons license, and indicate if changes were made. 
we study the nonlinear generalized Volterra integral equation given by

$$
y(x)=\kappa(x)+s(x) \int_{c}^{x} G(x, \tau, y(\tau)) d \tau, \quad \forall x \in I
$$

where $y: I \rightarrow E$ is unknown mapping.

Definition 1.1 We say that the nonlinear generalized Volterra integral equation (1.1) has the Hyers-Ulam stability, if for any $\epsilon>0$ and any mapping $\varphi: I \rightarrow E$ satisfying the inequality

$$
\left\|\varphi(x)-\kappa(x)-s(x) \int_{c}^{x} G(x, \tau, \varphi(\tau)) d \tau\right\| \leq \epsilon, \quad \forall x \in I
$$

there exists a solution mapping $y: I \rightarrow E$ of the integral equation (1.1) such that

$$
\|\varphi(x)-y(x)\| \leq \xi \epsilon, \quad \forall x \in I
$$

for some constant $\xi>0$.

In fact, (1.1) contains several important integral equations as special cases. For example, let $\lambda \in \mathcal{K}$ with $\lambda \neq 0$ and take $c:=a$. Define the mapping $s: I \rightarrow \mathcal{K}$ by

$$
s(x)=\lambda \quad \text { for all } x \in I .
$$

Then (1.1) will reduce to the following nonlinear Volterra integral equation studied by Akkouchi [5]:

$$
y(x)=\kappa(x)+\lambda \int_{a}^{x} G(x, \tau, y(\tau)) d \tau, \quad \forall x \in I .
$$

If we take $c:=a, E:=\mathbb{C}, s(x)=1$ for all $x \in I$ and let $\kappa$ be a zero function in (1.1), then (1.1) will reduce to the following nonlinear Volterra integral equation studied by Castro and Ramos [6]:

$$
y(x)=\int_{a}^{x} G(x, \tau, y(\tau)) d \tau, \quad \forall x \in I .
$$

Let $X$ be a nonempty set. Recall that a function $p: X \times X \rightarrow[0, \infty]$ is called a generalized metric [15-18] on $X$ (defined by Luxemburg [17]), if the following conditions hold:

(GM1) $p(x, y)=0$ if and only if $x=y$;

(GM2) $p(x, y)=p(y, x)$ for all $x, y \in X$;

(GM3) $p(x, z) \leq p(x, y)+p(y, z)$ for all $x, y, z \in X$.

The pair $(X, p)$ is then called a generalized metric space.

We remark that the only one difference of the generalized metric from the usual metric is that the range of the former is permitted to include the infinity. A generalized Banach contraction principle in a complete generalized metric space proved by Diaz and Margolis [15] has played an important role in the study of stability of functional equations. 
Theorem 1.1 (Diaz and Margolis [15]) Let $(X, p)$ be a complete generalized metric space and $T: X \rightarrow X$ be a selfmapping on $X$. Assume that there exists a nonnegative real number $\lambda<1$ such that

$$
p(T x, T y) \leq \lambda p(x, y) \quad \text { for all } x, y \in X
$$

Denote $T^{0}=I$, the identity mapping. Then, for a given element $u \in X$, exactly one of the following assertions is true:

(a) $p\left(T^{n} u, T^{n+1} u\right)=\infty$ for all $n \in \mathbb{N} \cup\{0\}$,

(b) there exists a nonnegative integer $\ell$ such that $p\left(T^{n} u, T^{n+1} u\right)<\infty$ for all $n \geq \ell$.

Actually, if the assertion (b) holds, then

(b1) the sequence $\left\{T^{n} u\right\}_{n \in \mathbb{N} \cup\{0\}}$ is convergent to a fixed point $\hat{y}$ of $T$;

(b2) $\hat{y}$ is the unique fixed point of $T$ in the set $\mathcal{S}$, where

$$
\mathcal{S}=\left\{x \in X: p\left(T^{\ell} u, x\right)<\infty\right\}
$$

(b3) $p(x, \hat{y}) \leq \frac{1}{1-\lambda} p(x, T x)$ for all $x \in \mathcal{S}$.

Let $T$ be a mapping with domain $D(T)$ and range $R(T)$ in a normed space $(E,\|\cdot\|)$. Recall that $T$ is said to be Lipschitzian (or to satisfy the Lipschitz condition) if there is a constant $L>0$ such that

$$
\|T x-T y\| \leq L\|x-y\| \quad \text { for all } x, y \in E .
$$

The smallest constant $L$ satisfying (1.2) is called the Lipschitz constant for $T$. It is known that the Lipschitz condition is very important for the study of the stability of functional equations. Till now, to the best of my knowledge, the Lipschitz condition with Lipschitz constant $L$ was almost assumed to satisfy $\gamma L<1$ for some positive real number $\gamma$ in the literature on the stability of functional equations. In this work, some weak conditions are utilized instead of the Lipschitz condition in the study of the stability of functional equations.

The main aim of this paper is the study of the existence theorem of the Hyers-Ulam stability for a general class of the nonlinear Volterra integral equations in Banach spaces. In Section 2, we first establish some properties for generalized metric spaces and present a generalization of Diaz-Margolis's fixed point theorem. As interesting applications of the generalized Diaz-Margolis fixed point theorem, we establish some existence theorems of the Hyers-Ulam stability for a general class of the nonlinear Volterra integral equations in Banach spaces in Section 3. Our new results improve and extend some known results in the literature.

\section{A generalization of Diaz-Margolis's fixed point theorem for $\mathcal{M T}$-functions}

In the present section, we shall establish a generalization of Diaz-Margolis's fixed point theorem (i.e. Theorem 1.1) for $\mathcal{M T}$-functions in the setting of complete generalized metric spaces. We may begin with the following definitions.

Definition 2.1 Let $(X, p)$ be a generalized metric space, $x \in X$ and $\left\{x_{n}\right\}_{n \in \mathbb{N}}$ a sequence in $X$. 
(i) $\left\{x_{n}\right\}$ is said to $p$-converge to $x$ if for any $\varepsilon>0$ there exists a natural number $n_{0}$ such that $p\left(x_{n}, x\right)<\varepsilon$ for all $n \geq n_{0}$. We denote this by $p-\lim _{n \rightarrow \infty} x_{n}=x$ or $x_{n} \stackrel{p}{\longrightarrow} x$ as $n \rightarrow \infty$ and call $x$ the limit of $\left\{x_{n}\right\}$.

(ii) $\left\{x_{n}\right\}$ is said to be a $p$-Cauchy sequence if for any $\varepsilon>0$ there exists a natural number $\mathbb{N}_{0}$ such that $p\left(x_{n}, x_{m}\right)<\varepsilon$ for all $n, m \geq \mathbb{N}_{0}$.

(iii) $(X, p)$ is said to be complete if every $p$-Cauchy sequence in $X$ is $p$-convergent.

Definition 2.2 Let $A$ be a nonempty subset of a generalized metric space $(X, p)$.

(i) The $p$-closure of $A$, denoted $c l_{p}(A)$, is defined by

$$
c l_{p}(A)=\left\{x \in X: \exists\left\{x_{n}\right\} \subset A \text { such that } x_{n} \stackrel{p}{\longrightarrow} x \text { as } n \rightarrow \infty\right\} .
$$

Obviously, $A \subseteq c l_{p}(A)$.

(ii) $A$ is said to be $p$-closed if $A=\operatorname{cl}_{p}(A)$.

(iii) $A$ is said to be $p$-open if the complement $X \backslash A$ of $A$ is $p$-closed.

Theorem 2.1 Let $(X, p)$ be a generalized metric space and let

$$
\mathcal{T}_{p}=\{U \subseteq X: U \text { is p-open in }(X, p)\}
$$

Then $\mathcal{T}_{p}$ is a topology on $(X, p)$ induced by $p$.

Proof It is obvious that $\emptyset$ and $X$ are $p$-closed in $(X, p)$. So $X$ and $\emptyset$ are $p$-open in $(X, p)$. Hence $\emptyset, X \in \mathcal{T}_{p}$. Let $U_{1}, U_{2} \in \mathcal{T}_{p}$. Then $V_{1}=X \backslash U_{1}$ and $V_{2}=X \backslash U_{2}$ are $p$-closed in $(X, p)$. We show $U_{1} \cap U_{2} \in \mathcal{T}_{p}$. Indeed, let $x \in l_{p}\left(V_{1} \cup V_{2}\right)$. Then there exists $\left\{x_{n}\right\} \subset V_{1} \cup V_{2}$ such that $x_{n} \stackrel{p}{\longrightarrow} x$ as $n \rightarrow \infty$. Without loss of generality, we may assume that there exists a subsequence $\left\{x_{n_{k}}\right\}$ of $\left\{x_{n}\right\} \cap V_{1}$. Since $x_{n_{k}} \stackrel{p}{\longrightarrow} x$ as $k \rightarrow \infty$, we get

$$
x \in c l_{p}\left(V_{1}\right)=V_{1} \subseteq V_{1} \cup V_{2} .
$$

So $c l_{p}\left(V_{1} \cup V_{2}\right) \subseteq V_{1} \cup V_{2}$ and hence $V_{1} \cup V_{2}$ is $p$-closed in $(X, p)$. Due to

$$
U_{1} \cap U_{2}=X \backslash\left(V_{1} \cup V_{2}\right),
$$

we know that $U_{1} \cap U_{2}$ is $p$-open in $(X, p)$. Hence $U_{1} \cap U_{2} \in \mathcal{T}_{p}$.

Let $I$ be any index set and let $\left\{U_{i}\right\}_{i \in I} \subset \mathcal{T}_{p}$. We verify $\bigcup_{i \in I} U_{i} \in \mathcal{T}_{p}$. For each $i \in I$, let $V_{i}=X \backslash U_{i}$. Thus $V_{i}$ is $p$-closed in $X$ for all $i \in I$. Let $z \in c l_{p}\left(\bigcap_{i \in I} V_{i}\right)$. Then there exists $\left\{z_{n}\right\} \subseteq \bigcap_{i \in I} V_{i}$ such that $z_{n} \stackrel{p}{\longrightarrow} z$ as $n \rightarrow \infty$. For each $i \in I$, since $\left\{z_{n}\right\} \subset V_{i}$ and $z_{n} \stackrel{p}{\longrightarrow} z$, we have

$$
z \in c l_{p}\left(V_{i}\right)=V_{i}
$$

Hence $z \in \bigcap_{i \in I} V_{i}$. So we get

$$
c l_{p}\left(\bigcap_{i \in I} V_{i}\right) \subseteq \bigcap_{i \in I} V_{i}
$$


which implies $\bigcap_{i \in I} V_{i}$ is $p$-closed in $(X, p)$. Since

$$
\bigcup_{i \in I} U_{i}=X \backslash \bigcap_{i \in I} V_{i}
$$

we know that $\bigcup_{i \in I} U_{i}$ is $p$-open in $(X, p)$ and hence $\bigcup_{i \in I} U_{i} \in \mathcal{T}_{p}$.

Therefore, from the above, we prove that $\mathcal{T}_{p}$ is a topology on $(X, p)$.

According to Theorem 2.1, we can give the definition of continuity of a mapping in generalized metric spaces. Actually, the definition of continuity can transfer essentially unchanged from classical metric spaces to generalized metric spaces as follows.

Definition 2.3 Let $\left(X, p_{X}\right)$ and $\left(Y, p_{X}\right)$ be generalized metric spaces and $\widehat{x} \in X$. A mapping $f: X \rightarrow Y$ is called continuous at $\widehat{x}$ if for any $\epsilon>0$, there exists a $\delta:=\delta(x, \epsilon)$ such that

$$
p_{Y}(f(x), f(\widehat{x}))<\epsilon \quad \text { whenever } x \in X \text { with } p_{X}(x, \widehat{x})<\delta .
$$

$f$ is called continuous on $X$ if $f$ is continuous at every point of $X$.

The following characterization of continuous functions can easily be verified.

Theorem 2.2 Let $\left(X, p_{X}\right)$ and $\left(Y, p_{Y}\right)$ be generalized metric spaces and $x_{0} \in X$. Then a mapping $f: X \rightarrow Y$ is continuous at $\widehat{x}$ if and only if $x_{n} \stackrel{p_{X}}{\longrightarrow} \widehat{x}$ implies $f\left(x_{n}\right) \stackrel{p_{Y}}{\longrightarrow} f(\widehat{x})$ as $n \rightarrow \infty$.

The following useful auxiliary result is crucial to our proofs.

Theorem 2.3 Let $(X, p)$ be a generalized metric space and $c \in X$. Let

$$
\mathcal{W}=\{x \in X: p(c, x)<\infty\}
$$

Define the function $f: \mathcal{W} \rightarrow[0, \infty)$ by

$$
f(x)=p(c, x) .
$$

Then the following statements hold:

(a) $p(u, v)<\infty$ for all $u, v \in \mathcal{W}$;

(b) $\mathcal{W}$ is $p$-closed in $(X, p)$;

(c) $|f(x)-f(y)| \leq p(x, y)$ for any $x, y \in \mathcal{W}$;

(d) $f$ is uniformly continuous on $\mathcal{W}$.

Proof Let $u, v \in \mathcal{W}$ be given. Then

$$
p(u, v) \leq p(u, c)+p(c, v)<\infty
$$

and hence (a) is proved. Next, we show (b). Let $a \in c l_{p}(\mathcal{W})$. Then there exists a sequence $\left\{a_{n}\right\} \subset \mathcal{W}$ such that $a_{n} \stackrel{p}{\longrightarrow} a$ as $n \rightarrow \infty$. So $p\left(c, a_{n}\right)<\infty$ for all $n \in \mathbb{N}$ and there exists a 
natural number $n_{0}$ such that $p\left(a_{n}, a\right)<1$ for all $n \geq n_{0}$. By (GM3), we have

$$
p(c, a) \leq p\left(c, a_{n_{0}}\right)+p\left(a_{n_{0}}, a\right)<p\left(c, a_{n_{0}}\right)+1<\infty
$$

which implies $a \in \mathcal{W}$. Thus $c l_{p}(\mathcal{W}) \subseteq \mathcal{W}$ and hence $\mathcal{W}$ is $p$-closed in $(X, p)$. To see (c), let $x, y \in \mathcal{W}$ be given. Then $f(x)=p(c, x)<\infty$ and $f(y)=p(c, y)<\infty$. By (GM2) and (GM3), we obtain

$$
p(c, x)-p(c, y) \leq p(y, x)=p(x, y) .
$$

Similarly,

$$
p(c, y)-p(c, x) \leq p(x, y)
$$

By (2.1) and (2.2), we get

$$
|f(x)-f(y)|=|p(c, x)-p(c, y)| \leq p(x, y) .
$$

Finally, we verify (d). Let $\epsilon>0$ be given. Take $\delta:=\epsilon$. Then for any $x, y \in \mathcal{W}$ with $p(x, y)<\delta$, by our conclusions (a) and (c), we have $|f(x)-f(y)|<\epsilon$. So $f$ is uniformly continuous on $\mathcal{W}$. The proof is completed.

Theorem 2.4 Let $(X, p)$ be a complete generalized metric space and $\mathcal{D}$ is a $p$-closed subset of $X$. Then $(\mathcal{D}, p)$ is also complete.

Proof Let $\left\{x_{n}\right\}$ be a $p$-Cauchy sequence in $\mathcal{D}$. By the completeness of $(X, p)$, there exists $v \in X$ such that $x_{n} \stackrel{p}{\longrightarrow} v$ as $n \rightarrow \infty$. By the $p$-closedness of $\mathcal{D}, v \in c l_{p}(\mathcal{D})=\mathcal{D}$. Hence we prove that $(\mathcal{D}, p)$ is complete.

Definition 2.4 [19-28] A function $\alpha:[0, \infty) \rightarrow[0,1)$ is said to be an $\mathcal{M T}$-function or $\mathcal{R}$-function if

$$
(*) \quad \limsup _{s \rightarrow t^{+}} \alpha(s)<1 \quad \text { for all } t \in[0, \infty)
$$

Remark 2.1 In fact, Reich used the property $(*)$ in [24]. In [24], p.40, he proved that a mapping $T: X \rightarrow K(X)$ has a fixed point in $X$ if it satisfies $\mathcal{H}(T x, T y) \leq \varphi(d(x, y)) d(x, y)$ for all $x, y \in X$ with $x \neq y$, where $K(X)$ denotes the family of all nonempty compact subsets of $X$ and $\varphi:(0, \infty) \rightarrow[0,1)$ satisfies $\lim _{\sup } \rightarrow t^{+} \varphi(s)<1$ for every $t \in(0, \infty)$. One of the conjectures made by Reich in $[25,26]$ asked whether or not the range of $T$ can be relaxed. In 1983, Reich posed the following famous open question [26] (see also [28]): Let $(X, d)$ be a complete metric space and $T: X \rightarrow \mathcal{C B}(X)$ be a multivalued mapping, where $\mathcal{C B}(X)$ denotes the family of all nonempty closed and bounded subsets of $X$. Suppose that

$$
\mathcal{H}(T x, T y) \leq \varphi(d(x, y)) d(x, y) \quad \text { for all } x, y \in X
$$

where $\mathcal{H}$ is the Hausdorff metric on $\mathcal{C B}(X)$ induced by the metric $d$ on $X$ and $\varphi:[0, \infty) \rightarrow$ $[0,1)$ satisfies the property $(*)$ except for $t=0$. Does $T$ have a fixed point? Mizoguchi 
and Takahashi were the first to give a partial answer to Reich's open question in 1989 (see [23]). A number of partial answers to Reich's open question have been investigated by many authors; see, e.g., [19-21, 23, 27, 28] and references therein.

It is obvious that if $\varphi:[0, \infty) \rightarrow[0,1)$ is a nondecreasing function or a nonincreasing function, then $\varphi$ is an $\mathcal{M T}$-function. So the set of $\mathcal{M T}$-functions is a rich class. In 2012, $\mathrm{Du}[20]$ established the following characterizations of $\mathcal{M T}$-functions.

Theorem 2.5 ([20], Theorem 2.1) Let $\varphi:[0, \infty) \rightarrow[0,1)$ be a function. Then the following statements are equivalent.

(a) $\varphi$ is an $\mathcal{M T}$-function.

(b) For each $t \in[0, \infty)$, there exist $r_{t}^{(1)} \in[0,1)$ and $\varepsilon_{t}^{(1)}>0$ such that $\varphi(s) \leq r_{t}^{(1)}$ for all $s \in\left(t, t+\varepsilon_{t}^{(1)}\right)$.

(c) For each $t \in[0, \infty)$, there exist $r_{t}^{(2)} \in[0,1)$ and $\varepsilon_{t}^{(2)}>0$ such that $\varphi(s) \leq r_{t}^{(2)}$ for all $s \in\left[t, t+\varepsilon_{t}^{(2)}\right]$.

(d) For each $t \in[0, \infty)$, there exist $r_{t}^{(3)} \in[0,1)$ and $\varepsilon_{t}^{(3)}>0$ such that $\varphi(s) \leq r_{t}^{(3)}$ for all $s \in\left(t, t+\varepsilon_{t}^{(3)}\right]$.

(e) For each $t \in[0, \infty)$, there exist $r_{t}^{(4)} \in[0,1)$ and $\varepsilon_{t}^{(4)}>0$ such that $\varphi(s) \leq r_{t}^{(4)}$ for all $s \in\left[t, t+\varepsilon_{t}^{(4)}\right)$.

(f) For any nonincreasing sequence $\left\{x_{n}\right\}_{n \in \mathbb{N}}$ in $[0, \infty)$, we have $0 \leq \sup _{n \in \mathbb{N}} \varphi\left(x_{n}\right)<1$.

(g) $\varphi$ is a function of contractive factor; that is, for any strictly decreasing sequence $\left\{x_{n}\right\}_{n \in \mathbb{N}}$ in $[0, \infty)$, we have $0 \leq \sup _{n \in \mathbb{N}} \varphi\left(x_{n}\right)<1$.

The main result of this section is formulated in the following new fixed theorem in complete generalized metric spaces, which generalize and improve Diaz-Margolis's fixed point theorem.

Theorem 2.6 Let $(X, p)$ be a complete generalized metric space and $T: X \rightarrow X$ be a selfmapping on $X$. Assume that there exists an $\mathcal{M T}$-function $\alpha:[0, \infty) \rightarrow[0,1)$ such that

$$
p(T x, T y) \leq \alpha(p(x, y)) p(x, y) \quad \text { for all } x, y \in X \text { with } p(x, y)<\infty
$$

Denote $T^{0}=I$, the identity mapping. Then, for a given element $u \in X$, exactly one of the following assertions is true:

(a) $p\left(T^{n} u, T^{n+1} u\right)=\infty$ for all $n \in \mathbb{N} \cup\{0\}$;

(b) there exists a nonnegative integer $\ell$ such that $p\left(T^{n} u, T^{n+1} u\right)<\infty$ for all $n \geq \ell$.

Actually, if the assertion (b) holds, then

(b1) the sequence $\left\{T^{n} u\right\}_{n \in \mathbb{N} \cup\{0\}}$ is convergent to a fixed point $v$ of $T$;

(b2) $v$ is the unique fixed point of $T$ in the set $\mathcal{L}$, where

$$
\mathcal{L}=\left\{x \in X: p\left(T^{\ell} u, x\right)<\infty\right\}
$$

(b3) $p(x, v) \leq \frac{1}{1-\alpha(p(x, v))} p(x, T x)$ for all $x \in \mathcal{L}$.

Proof Let $u \in X$ be given. Define $x_{0}=u$ and $x_{n}=T x_{n-1}=T^{n} u$ for each $n \in \mathbb{N}$. Suppose that (a) does not hold. Then there exists a nonnegative integer $\ell$ such that

$$
p\left(x_{\ell}, x_{\ell+1}\right)=p\left(T^{\ell} u, T^{\ell+1} u\right)<\infty .
$$


By (2.3), we have

$$
p\left(x_{\ell+1}, x_{\ell+2}\right)=p\left(T x_{\ell}, T x_{\ell+1}\right) \leq \alpha\left(p\left(x_{\ell}, x_{\ell+1}\right)\right) p\left(x_{\ell}, x_{\ell+1}\right)<p\left(x_{\ell}, x_{\ell+1}\right)<\infty .
$$

So, it follows from (2.4) and (2.5) that

$$
p\left(x_{\ell}, x_{\ell+2}\right) \leq p\left(x_{\ell}, x_{\ell+1}\right)+p\left(x_{\ell+1}, x_{\ell+2}\right)<\infty .
$$

Let $w_{n}=x_{n+\ell-1}$ for each $n \in \mathbb{N}$. Then $w_{1}=x_{\ell}=T^{\ell} u$. From (2.4), (2.5), and (2.6), we have $p\left(w_{1}, w_{2}\right), p\left(w_{2}, w_{3}\right)<\infty$ and

$$
w_{1}, w_{2}, w_{3} \in \mathcal{L}=\left\{x \in X: p\left(w_{1}, x\right)<\infty\right\}
$$

By induction, we obtain, for any $n \in \mathbb{N}$ :

(i) $w_{n} \in \mathcal{L}$,

(ii) $p\left(w_{n}, w_{n+1}\right)<\infty$,

(iii) $p\left(w_{n+1}, w_{n+2}\right) \leq \alpha\left(p\left(w_{n}, w_{n+1}\right)\right) p\left(w_{n}, w_{n+1}\right)$.

From (ii), we obtain the conclusion (b). We now verify that (b1), (b2), and (b3) are true. By (iii), we know that $\left\{p\left(w_{n}, w_{n+1}\right)\right\}_{n \in \mathbb{N}}$ is a strictly decreasing sequence in $[0, \infty)$. Since $\alpha$ is an $\mathcal{M T}$-function, by (g) of Theorem 2.5 , we have

$$
0 \leq \sup _{n \in \mathbb{N}} \alpha\left(p\left(w_{n}, w_{n+1}\right)\right)<1
$$

Let $\gamma:=\sup _{n \in \mathbb{N}} \alpha\left(p\left(w_{n}, w_{n+1}\right)\right)$. So $\gamma \in[0,1)$. By (iii) again, we get

$$
\begin{aligned}
p\left(w_{n+1}, w_{n+2}\right) & <\alpha\left(p\left(w_{n}, w_{n+1}\right)\right) p\left(w_{n}, w_{n+1}\right) \\
& \leq \gamma p\left(w_{n}, w_{n+1}\right) \\
& <\gamma^{2} p\left(w_{n-1}, w_{n}\right) \\
& <\cdots \\
& <\gamma^{n} p\left(w_{1}, w_{2}\right) \text { for each } n \in \mathbb{N} .
\end{aligned}
$$

Let $\lambda_{n}=\frac{\gamma^{n-1}}{1-\gamma} p\left(w_{1}, w_{2}\right), n \in \mathbb{N}$. For $m>n$ with $m, n \in \mathbb{N}$, by (2.7), we get

$$
p\left(w_{n}, w_{m}\right) \leq \sum_{j=n}^{m-1} p\left(w_{j}, w_{j+1}\right)<\lambda_{n} .
$$

Since $\gamma \in[0,1)$, we obtain $\lim _{n \rightarrow \infty} \lambda_{n}=0$ and hence $\lim _{n \rightarrow \infty} \sup \left\{p\left(w_{n}, w_{m}\right): m>n\right\}=0$. So, $\left\{w_{n}\right\}_{n \in \mathbb{N}}$ is a $p$-Cauchy sequence in $\mathcal{L}$. Applying Theorems 2.3 and 2.4, we conclude that $(\mathcal{L}, p)$ is also a complete generalized metric space. So, there exists $v \in \mathcal{L}$ such that $w_{n} \stackrel{p}{\longrightarrow} v$ as $n \rightarrow \infty$.

We now show that $v \in \mathcal{F}(T)$. Let

$$
\mathcal{W}=\{x \in X: p(v, x)<\infty\}
$$


Clearly, $v \in \mathcal{W}$. Note that $v, w_{n} \in \mathcal{L}$ implies $w_{n} \in \mathcal{W}$ for all $n \in \mathbb{N}$. For each $n \in \mathbb{N}$, we obtain

$$
\begin{aligned}
p(v, T v) & \leq p\left(v, w_{n+1}\right)+p\left(w_{n+1}, T v\right) \\
& \leq p\left(v, w_{n+1}\right)+\alpha\left(p\left(w_{n}, v\right)\right) p\left(w_{n}, v\right) \\
& <p\left(v, w_{n+1}\right)+p\left(w_{n}, v\right) .
\end{aligned}
$$

Applying Theorem 2.3 again, we know that the function $x \mapsto p(v, x)$ is continuous on the set $\mathcal{W}$. So, by taking the limit at both sides of the previous inequality and applying Theorem 2.2, we get $p(v, T v)=0$ or $T v=v$.

Next, we want to show the uniqueness of fixed point of $T$ in $\mathcal{L}$ (i.e. $\mathcal{F}(T) \cap \mathcal{L}$ is a singleton set). We have shown $v \in \mathcal{F}(T) \cap \mathcal{L}$, so it suffices to show that $\mathcal{F}(T) \cap \mathcal{L}=\{v\}$. Let $z \in$ $\mathcal{F}(T) \cap \mathcal{L}$. Since $z, v \in \mathcal{L}$, we know $p(z, v)<\infty$ from Theorem 2.3. By (2.3), we obtain

$$
p(z, v)=p(T z, T v) \leq \alpha(p(z, v)) p(z, v)
$$

which implies

$$
(1-\alpha(p(z, v))) p(z, v) \leq 0
$$

Since $\alpha(p(z, v)) \in[0,1)$, from the last inequality one deduces $p(z, v)=0$ or $z=v$. So we must have $\mathcal{F}(T) \cap \mathcal{L}=\{v\}$.

Finally, we verify the inequality $p(x, v) \leq \frac{1}{1-\alpha(p(x, v))} p(x, T x)$ for all $x \in \mathcal{L}$. Let $x \in \mathcal{L}$ be given. Since $v \in \mathcal{L}$, we know $p(x, v)<\infty$. By $(2.3)$, we have

$$
p(T x, T v) \leq \alpha(p(x, v)) p(x, v) .
$$

Since

$$
\begin{aligned}
p(x, v)-\alpha(p(x, v)) p(x, v) & \leq p(x, v)-p(T x, T v) \\
& =p(x, v)-p(T x, v) \\
& \leq p(x, T x)
\end{aligned}
$$

we deduce

$$
p(x, v) \leq \frac{1}{1-\alpha(p(x, v))} p(x, T x) .
$$

The proof is completed.

\section{Existence of the Hyers-Ulam stability for generalized Volterra integral equations}

In this section, we study the existence theorem of the Hyers-Ulam stability for a general class of the nonlinear Volterra integral equations in Banach spaces by applying Theorem 2.6.

Theorem 3.1 Let $(E,\|\cdot\|)$ be a Banach space over a field $\mathcal{K}$ (either $\mathbb{R}$ or $\mathbb{C}$ ). Let $a$ and $b$ be given real numbers with $a<b$ and let $I=[a, b]$. Let $c \in I$ and let $\mu: \mathbb{R} \rightarrow \mathbb{R}$ be a function 
and $: I \rightarrow \mathcal{K}$ be a continuous mapping with $\max _{x \in I}|s(x)|:=\lambda>0$. Assume that the function $\mu: \mathbb{R} \rightarrow \mathbb{R}$ is nondecreasing on $[0, \infty)$ satisfying

$$
\mu([0, \infty)) \subseteq\left[0, \frac{\delta}{\lambda(b-a)}\right]
$$

for some constant $0<\delta<1$, and $G: I \times I \times E \rightarrow E$ is a continuous mapping satisfying

$$
\|G(x, \tau, y)-G(x, \tau, z)\| \leq \mu(\|y-z\|)\|y-z\| \quad \text { for any } x, \tau \in I \text { and } y, z \in E \text {. }
$$

If there exist two continuous mappings $\varphi, \kappa: I \rightarrow E$ satisfying

$$
\left\|\varphi(x)-\kappa(x)-s(x) \int_{c}^{x} G(x, \tau, \varphi(\tau)) d \tau\right\| \leq \epsilon
$$

for each $x \in I$ and some constant $\epsilon \geq 0$, then there exists a unique continuous mapping $y: I \rightarrow$ Esuch that

$$
y(x)=\kappa(x)+s(x) \int_{c}^{x} G(x, \tau, y(\tau)) d \tau
$$

and

$$
\|\varphi(x)-y(x)\| \leq \frac{\epsilon}{1-\delta}
$$

for all $x \in I$.

Proof Let $X:=\mathcal{C}(I, E)$ denote the set of all continuous functions from $I$ to $E$. Define a function $\widehat{p}: X \times X \rightarrow[0, \infty]$ by

$$
\widehat{p}(f, g)=\inf \{M \geq 0:\|f(x)-g(x)\| \leq M \text { for all } x \in I\}
$$

where we adopt the usual convention that $\inf \emptyset=\infty$. Clearly, $\|f(x)-g(x)\| \leq \widehat{p}(f, g)$ for all $x \in I$. Following a similar argument as in the proof of [7], Theorem 2.1, or [8], Theorem 3.1, one can verify that $(X, \widehat{p})$ is a complete generalized metric space. Let us now introduce the operator $T: X \rightarrow X$, which is defined by

$$
(T f)(x)=\kappa(x)+s(x) \int_{c}^{x} G(x, \tau, f(\tau)) d \tau
$$

for all $f \in X$ and $x \in I$. Then $T f \in X$ for all $f \in X$. Indeed, let $f \in X$ be given. For any $x, x_{0} \in I$, since $\max _{x \in I}|s(x)|=\lambda$, we have

$$
\begin{aligned}
& \left\|(T f)(x)-(T f)\left(x_{0}\right)\right\| \\
& \quad=\left\|\kappa(x)+s(x) \int_{c}^{x} G(x, \tau, \varphi(\tau)) d \tau-\kappa\left(x_{0}\right)-s\left(x_{0}\right) \int_{c}^{x_{0}} G\left(x_{0}, \tau, f(\tau)\right) d \tau\right\| \\
& \quad \leq\left\|\kappa(x)-\kappa\left(x_{0}\right)\right\|+|s(x)|\left\|\int_{c}^{x} G(x, \tau, f(\tau)) d \tau-\int_{c}^{x} G\left(x_{0}, \tau, f(\tau)\right) d \tau\right\|
\end{aligned}
$$




$$
\begin{aligned}
& +\left|s(x)-s\left(x_{0}\right)\right|\left\|\int_{c}^{x} G\left(x_{0}, \tau, f(\tau)\right) d \tau\right\| \\
& +\left|s\left(x_{0}\right)\right|\left\|\int_{c}^{x} G\left(x_{0}, \tau, f(\tau)\right) d \tau-\int_{c}^{x_{0}} G\left(x_{0}, \tau, f(\tau)\right) d \tau\right\| \\
& \leq\left\|\kappa(x)-\kappa\left(x_{0}\right)\right\|+\lambda\left|\int_{c}^{x}\left\|G(x, \tau, f(\tau))-G\left(x_{0}, \tau, f(\tau)\right)\right\| d \tau\right| \\
& +\left|s(x)-s\left(x_{0}\right)\right|\left\|\int_{c}^{x} G\left(x_{0}, \tau, f(\tau)\right) d \tau\right\|+\lambda\left\|\int_{x_{0}}^{x} G\left(x_{0}, \tau, f(\tau)\right) d \tau\right\| .
\end{aligned}
$$

Since $s, \kappa, G$, and $f$ are continuous, the last inequality implies that

$$
(T f)(x) \rightarrow(T f)\left(x_{0}\right) \quad \text { as } x \rightarrow x_{0} .
$$

So $T f$ is continuous and hence $T f \in X$ for all $f \in X$.

Now, we claim that there exists an $\mathcal{M T}$-function $\alpha:[0, \infty) \rightarrow[0,1)$ such that

$$
\widehat{p}(T f, T g) \leq \alpha(\widehat{p}(f, g)) \widehat{p}(f, g) \quad \text { for all } f, g \in X \text { with } \widehat{p}(f, g)<\infty
$$

Indeed, according to the inequality (3.1) and the function $\mu$ is nondecreasing on $[0, \infty)$, we can define an $\mathcal{M T}$-function $\alpha:[0, \infty) \rightarrow[0,1)$ by

$$
\alpha(t)=\lambda(b-a) \mu(t)
$$

Let $f, g \in X$ with $\widehat{p}(f, g)<\infty$. Given $\varepsilon>0$. Since

$$
\widehat{p}(f, g)<\widehat{p}(f, g)+\frac{\varepsilon}{1+\alpha(\widehat{p}(f, g))},
$$

there exists $M_{f g} \geq 0$ such that

$$
M_{f g}<\widehat{p}(f, g)+\frac{\varepsilon}{1+\alpha(\widehat{p}(f, g))}
$$

and

$$
\|f(x)-g(x)\| \leq M_{f g} \quad \text { for all } x \in I
$$

On the other hand, since $\mu$ is nondecreasing on $[0, \infty)$ and $\|f(x)-g(x)\| \leq \widehat{p}(f, g)$ for all $x \in I$, we have

$$
\mu(\|f(x)-g(x)\|) \leq \mu(\widehat{p}(f, g)) \quad \text { for all } x \in I .
$$

For any $x \in I$, by taking into account (3.2), (3.4), (3.5), (3.6), (3.7), and (3.8), we get

$$
\begin{aligned}
\|(T f)(x)-(T g)(x)\| & =|s(x)|\left\|\int_{c}^{x}(G(x, \tau, f(\tau))-G(x, \tau, g(\tau))) d \tau\right\| \\
& \leq \lambda\left|\int_{c}^{x} \mu(\|f(\tau)-g(\tau)\|)\|f(\tau)-g(\tau)\| d \tau\right|
\end{aligned}
$$




$$
\begin{aligned}
& \leq \lambda \mu(\widehat{p}(f, g))\left|\int_{c}^{x}\|f(\tau)-g(\tau)\| d \tau\right| \\
& \leq \lambda(b-a) \mu(\widehat{p}(f, g)) M_{f g} \\
& <\alpha(\widehat{p}(f, g))\left(\widehat{p}(f, g)+\frac{\varepsilon}{1+\alpha(\widehat{p}(f, g))}\right) \\
& <\alpha(\widehat{p}(f, g)) \widehat{p}(f, g)+\varepsilon .
\end{aligned}
$$

Hence $\widehat{p}(T f, T g) \leq \alpha(\widehat{p}(f, g)) \widehat{p}(f, g)+\varepsilon$. Since $\varepsilon$ is arbitrary, we can conclude that

$$
\widehat{p}(T f, T g) \leq \alpha(\widehat{p}(f, g)) \widehat{p}(f, g) .
$$

Next, we prove that $\widehat{p}(T f, f)<\infty$ for all $f \in X$. Let $f \in X$ be given. Since $T f \in X$, we know that the function $x \mapsto\|(T f)(x)-f(x)\|$ is continuous on $I$. Then there exists a constant $M_{0} \geq 0$ such that

$$
\|(T f)(x)-f(x)\| \leq M_{0} \quad \text { for all } x \in I .
$$

From the last inequality one deduces that $\widehat{p}(T f, f) \leq M_{0}<\infty$.

Take $h \in X$. Then $\widehat{p}(T h, h)<\infty$. We will now verify that

$$
\{f \in X: \widehat{p}(h, f)<\infty\}=X \text {. }
$$

Indeed, it suffices to show that $X \subseteq\{f \in X: \widehat{p}(h, f)<\infty\}$. For any $f \in X$, since $f$ and $h$ are continuous on $I$, there exists a constant $\gamma \geq 0$ such that

$$
\|h(x)-f(x)\| \leq \gamma \quad \text { for any } x \in I
$$

which implies $\widehat{p}(h, f) \leq \gamma<\infty$. Hence we prove

$$
X \subseteq\{f \in X: \widehat{p}(h, f)<\infty\}
$$

Applying Theorem 2.6(b), there exists a unique $y \in X$ (that is, $y: I \rightarrow E$ is a continuous funtion) such that

$$
\begin{aligned}
& T^{n} h \stackrel{\widehat{p}}{\longrightarrow} y \quad \text { as } n \rightarrow \infty, \\
& T y=y,
\end{aligned}
$$

and

$$
\widehat{p}(f, y) \leq \frac{1}{1-\alpha(\widehat{p}(f, y))} \widehat{p}(f, T f) \quad \text { for all } f \in X .
$$

From (3.9), we have

$$
y(x)=\kappa(x)+s(x) \int_{c}^{x} G(x, \tau, y(\tau)) d \tau \quad \text { for all } x \in I
$$


By (3.3), we get

$$
\widehat{p}(\varphi, T \varphi) \leq \epsilon
$$

Since $\mu(\widehat{p}(\varphi, y)) \leq \frac{\delta}{\lambda(b-a)}$, by taking into account (3.10), (3.11), and the last inequality, we obtain

$$
\widehat{p}(\varphi, y) \leq \frac{1}{1-\alpha(\widehat{p}(\varphi, y))} \widehat{p}(\varphi, T \varphi) \leq \frac{\epsilon}{1-\delta}
$$

which implies

$$
\|\varphi(x)-y(x)\| \leq \frac{\epsilon}{1-\delta} \quad \text { for all } x \in I .
$$

The proof is completed.

The following conclusions are immediately drawn from Theorem 3.1.

Corollary 3.1 Let $(E,\|\cdot\|)$ be a Banach space over a field $\mathcal{K}$ (either $\mathbb{R}$ or $\mathbb{C})$. Let a and $b$ be given real numbers with $a<b$ and let $I=[a, b]$. Let $c \in I$ and let $s: I \rightarrow \mathcal{K}$ be a continuous mapping with $\max _{x \in I}|s(x)|:=\lambda>0$. Let L be a positive constant with $0<\lambda L(b-a)<1$. Let $c: I \rightarrow \mathcal{K}$ be a continuous mapping. Assume that $G: I \times I \times E \rightarrow E$ is a continuous mapping which satisfies the following Lipschitz condition:

$$
\|G(x, \tau, y)-G(x, \tau, z)\| \leq L\|y-z\| \quad \text { for any } x, \tau \in I \text { and } y, z \in E .
$$

If there exist two continuous mappings $\varphi, \kappa: I \rightarrow$ E satisfying

$$
\left\|\varphi(x)-\kappa(x)-s(x) \int_{c}^{x} G(x, \tau, \varphi(\tau)) d \tau\right\| \leq \epsilon
$$

for each $x \in I$ and some constant $\epsilon \geq 0$, then there exists a unique continuous mapping $y: I \rightarrow$ Esuch that

$$
y(x)=\kappa(x)+s(x) \int_{c}^{x} G(x, \tau, y(\tau)) d \tau
$$

and

$$
\|\varphi(x)-y(x)\| \leq \frac{\epsilon}{1-\lambda L(b-a)}
$$

for all $x \in I$.

Proof Let $V: \mathbb{R} \rightarrow \mathbb{R}$ be any function. Define $\mu: \mathbb{R} \rightarrow \mathbb{R}$ by

$$
\mu(t)= \begin{cases}L, & \text { for } t \geq 0 \\ V(t), & \text { otherwise }\end{cases}
$$


Put $\delta:=\lambda L(b-a)$. Then $\mu$ is nondecreasing on $[0, \infty)$, satisfying

$$
\mu([0, \infty)) \subseteq\left[0, \frac{\delta}{\lambda(b-a)}\right]
$$

So one can get the thesis by applying Theorem 3.1 .

\section{Remark 3.1}

(a) Corollary 3.1 actually implies Theorem 3.1. Indeed, under the hypotheses of Theorem 3.1, we set $L:=\frac{\delta}{\lambda(b-a)}$. Due to (3.1), (3.2), and $0<\delta<1$, we get the following:

- $\|G(x, \tau, y)-G(x, \tau, z)\| \leq L\|y-z\|$ for any $x, \tau \in I$ and $y, z \in E$;

- $0<\lambda L(b-a)<1$.

So all the hypotheses of Corollary 3.1 are fulfilled. It is therefore possible to apply Corollary 3.1 to get the conclusion of Theorem 3.1.

(b) [5], Theorem 3.1, and [6], Theorem 5.1, are special cases of Theorem 3.1.

Corollary 3.2 Let $(E,\|\cdot\|)$ be a Banach space over a field $\mathcal{K}($ either $\mathbb{R}$ or $\mathbb{C})$ and $\zeta \in \mathcal{K}$ with $\zeta \neq 0$. Let $a$ and $b$ be given real numbers with $a<b$ and let $I=[a, b]$. Let $c \in I$. Assume that the function $\mu: \mathbb{R} \rightarrow \mathbb{R}$ is nondecreasing on $[0, \infty)$ satisfying

$$
\mu([0, \infty)) \subseteq\left[0, \frac{\delta}{|\zeta|(b-a)}\right]
$$

for some constant $0<\delta<1$, and $G: I \times I \times E \rightarrow E$ is a continuous mapping satisfying

$$
\|G(x, \tau, y)-G(x, \tau, z)\| \leq \mu(\|y-z\|)\|y-z\| \quad \text { for any } x, \tau \in I \text { and } y, z \in E \text {. }
$$

If there exist two continuous mappings $\varphi, \kappa: I \rightarrow$ E satisfying

$$
\left\|\varphi(x)-\kappa(x)-\zeta \int_{c}^{x} G(x, \tau, \varphi(\tau)) d \tau\right\| \leq \epsilon
$$

for each $x \in I$ and some constant $\epsilon \geq 0$, then there exists a unique continuous mapping $y: I \rightarrow$ Esuch that

$$
y(x)=\kappa(x)+\zeta \int_{c}^{x} G(x, \tau, y(\tau)) d \tau
$$

and

$$
\|\varphi(x)-y(x)\| \leq \frac{\epsilon}{1-\delta}
$$

for all $x \in I$.

Proof Define a continuous function $s: I \rightarrow \mathcal{K}$ by

$$
s(x)=\zeta \quad \text { for all } x \in I
$$

Thus $\lambda:=\max _{x \in I}|s(x)|=|\zeta|>0$. Therefore the desired conclusion follows from Theorem 3.1 immediately. 
Remark 3.2 Recently, Jung et al. obtained an interesting result on Hyers-Ulam stability of the linear functional equation in a single variable $f(\phi(x))=g(x) \cdot f(x)$ on a complete metric group (for more details, see [10]). The results in this paper can be generalized further in the spirit of complete metric groups as in [10].

\section{Competing interests}

The author declares that he has no competing interests.

\section{Acknowledgements}

The author wishes to express his cordial thanks to Professor Simeon Reich and the anonymous referees for their valuable suggestions and comments. The author was supported by Grant No. MOST 103-2115-M-017-001 and Grant No. MOST 104-2115-M-017-002 of the Ministry of Science and Technology of the Republic of China.

Received: 16 June 2015 Accepted: 6 December 2015 Published online: 22 December 2015

\section{References}

1. Ulam, SM: A Collection of Mathematical Problems. Interscience Tracts in Pure and Applied Mathematics, vol. 8. Interscience, New York (1960)

2. Hyers, DH: On the stability of the linear functional equation. Proc. Natl. Acad. Sci. USA 27, $222-224$ (1941)

3. Aoki, T: On the stability of the linear transformation in Banach spaces. J. Math. Soc. Jpn. 2, 64-66 (1950)

4. Rassias, THM: On the stability of the linear mapping in Banach spaces. Proc. Am. Math. Soc. 72, $297-300$ (1978)

5. Akkouchi, M: Hyers-Ulam-Rassias stability of nonlinear Volterra integral equations via a fixed point approach. Acta Univ. Apulensis 26, 257-266 (2011)

6. Castro, LP, Ramos, A: Hyers-Ulam-Rassias stability for a class of nonlinear Volterra integral equations. Banach J. Math. Anal. 3(1), 36-43 (2009)

7. Jung, S-M: A fixed point approach to the stability of a Volterra integral equation. Fixed Point Theory Appl. 2007, Article ID 57064 (2007)

8. Jung, S-M: A fixed point approach to the stability of differential equations $y^{\prime}=F(x, y)$. Bull. Malays. Math. Soc. 33(1), 47-56 (2010)

9. Jung, S-M: Hyers-Ulam-Rassias Stability of Functional Equations in Nonlinear Analysis. Springer Optimization and Its Applications, vol. 48. Springer, New York (2011)

10. Jung, S-M, Popa, D, Rassias, MT: On the stability of the linear functional equation in a single variable on complete metric groups. J. Glob. Optim. 59, 165-171 (2014)

11. Jung, S-M, Rassias, MT, Mortici, C: On a functional equation of trigonometric type. Appl. Math. Comput. 252, 294-303 (2015)

12. Lakshmikantham, V, Rao, MRM: Theory of Integro-Differential Equations. Stability and Control: Theory, Methods and Applications, vol. 1. Gordon \& Breach, Philadelphia (1995)

13. Mortici, C, Rassias, MT, Jung, S-M: On the stability of a functional equation associated with the Fibonacci numbers Abstr. Appl. Anal. 2014, Article ID 546046 (2014)

14. Rus, IA: Ulam stability of the operatorial equations. In: Functional Equations in Mathematical Analysis. Springer Optimization and Its Applications, vol. 52, pp. 287-305. Springer, New York (2012)

15. Diaz, JB, Margolis, B: A fixed point theorem of the alternative, for contractions on a generalized complete metric space. Bull. Am. Math. Soc. 74, 305-309 (1968)

16. Jung, CFK: On generalized complete metric spaces. Bull. Am. Math. Soc. 75, 113-116 (1969)

17. Luxemburg, WAJ: On the convergence of successive approximations in the theory of ordinary differential equations. Indag. Math. 20, 540-546 (1958)

18. Petruşel, A, Rus, IA, Şerban, MA: Fixed points for operators on generalized metric spaces. CUBO 10, 45-66 (2008)

19. Du, W-S: Some new results and generalizations in metric fixed point theory. Nonlinear Anal. 73, 1439-1446 (2010)

20. Du, W-S: On coincidence point and fixed point theorems for nonlinear multivalued maps. Topol. Appl. 159, 49-56 (2012)

21. Du, W-S: On generalized weakly directional contractions and approximate fixed point property with applications. Fixed Point Theory Appl. 2012, Article ID 6 (2012)

22. Du, W-S: On Caristi-type mappings without lower semicontinuity assumptions. J. Fixed Point Theory Appl. 17 733-752 (2015)

23. Mizoguchi, N, Takahashi, W: Fixed point theorems for multivalued mappings on complete metric spaces. J. Math. Anal. Appl. 141, 177-188 (1989)

24. Reich, S: Fixed points of contractive functions. Boll. Unione Mat. Ital. 5, 26-42 (1972)

25. Reich, S: Some fixed point problems. Atti Accad. Naz. Lincei, Rend. Cl. Sci. Fis. Mat. Nat. (8) 57, 194-198 (1974)

26. Reich, S: Some problems and results in fixed point theory. Contemp. Math. 21, 179-187 (1983)

27. Reich, S, Zaslavski, AJ: Genericity in Nonlinear Analysis. Springer, New York (2014)

28. Xu, H-K: Metric fixed point theory for multivalued mappings. Diss. Math. 389, 1-39 (2000) 